\title{
Shaping Disk Galaxy Stellar Populations via Internal and External Processes
}

\author{
Rok Roškar \\ Institute for Theoretical Physics, University of Zürich, 8057 Zürich, Switzerland \\ email: roskar@physik.uzh.ch
}

\begin{abstract}
In recent years, effects such as the radial migration of stars in disks have been recognized as important drivers of the properties of stellar populations. Radial migration arises due to perturbative effects of disk structures such as bars and spiral arms, and can deposit stars formed in disks to regions far from their birthplaces. Migrant stars can significantly affect the demographics of their new locales, especially in low-density regions such as in the outer disks. However, in the cosmological environment, other effects such as mergers and filamentary gas accretion also influence the disk formation process. Understanding the relative importance of these processes on the detailed evolution of stellar population signatures is crucial for reconstructing the history of the Milky Way and other nearby galaxies. In the Milky Way disk in particular, the formation of the thickened component has recently attracted much attention due to its potential to serve as a diagnostic of the galaxy's early history. Some recent work suggests, however, that the vertical structure of Milky Way stellar populations is consistent with models that build up the thickened component through migration. I discuss these developments in the context of cosmological galaxy formation.
\end{abstract}
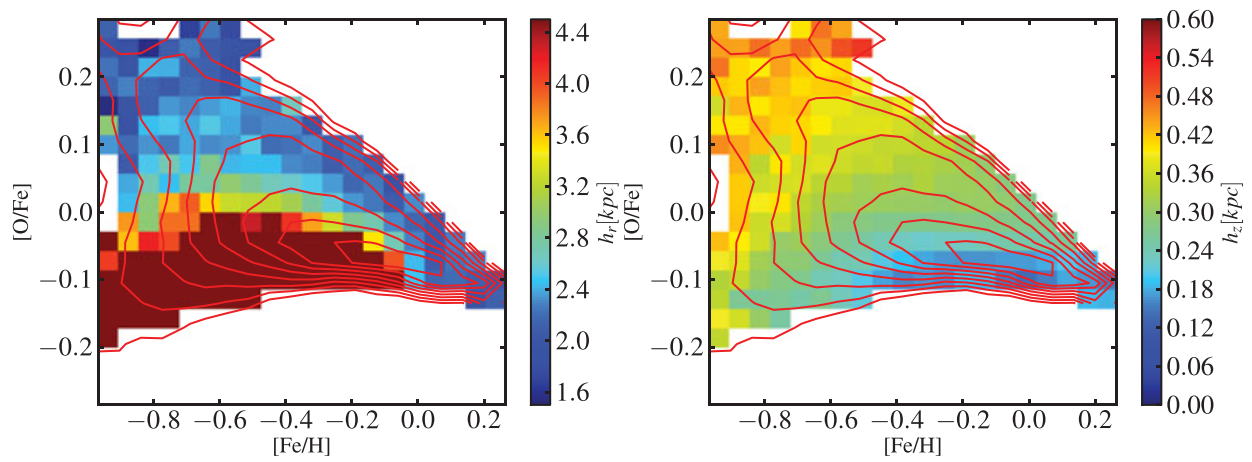

Figure 1. The simulated distribution of stars in the $[\mathrm{O} / \mathrm{Fe}]$ vs. $[\mathrm{Fe} / \mathrm{H}]$ plane, coloured by scale length $h_{r}$ on the left and scale height $h_{z}$ on the right (from Roškar 2012). The stars lie between $4<R$ [kpc] $<9$ and $|z|<3$ [kpc]. The smooth transition of the structural parameters in the abundance-metallicity plane is in broad agreement with the results of Bovy et al. 2012.

\section{References}

Bovy J., et al. 2012, ApJ, 753, 148

Roškar R. et al. 2008, ApJL, 675, L65

Roškar R., et al. 2010, MNRAS, 408, 783

Roškar R., Debattista V. P., \& Loebman S. R. 2012, arXiv 1211.1982 